\title{
A UNIQUENESS RESULT IN CONFORMAL MAPPING
}

\author{
JAMES A. JENKINS
}

1. Let $\Sigma$ denote the family of functions $f(z)$ meromorphic and univalent in $|z|>1$ with Laurent expansion in the neighborhood of the point at infinity given by

$$
f(z)=z+c_{0}+c_{1} / z+\cdots+c_{n} / z^{n}+\cdots .
$$

It is well known that, if $f$ belongs to the family $\Sigma$ and $E$ denotes the complement of the image of $|z|>1$ under the mapping $w=f(z)$, the diameter of $E$ belongs to the interval [2, 4]. The upper limit corresponds to the classical diameter theorem, obtained by elementary methods which automatically prove that equality is attained only for the slit mappings $f(z)=z+e^{i \alpha} z^{-1}+c, \alpha$ real, $c$ constant. The proof that the diameter is at least 2 is also fairly elementary, indicated for example in [5, IV Abschn., No. 141]. However this proof does not in any evident manner provide a complete uniqueness statement. While the assertion appears in the literature, for example in the reference just given, that equality is attained only for the functions $f(z)=z+c, c$ constant, the author has found no published proof of this statement. The object of this paper is to provide such a proof.

2. Theorem. If $f \in \Sigma$ and $E$, the complement of the image of $|z|>1$ under the mapping $w=f(z)$, has diameter 2 , then $f(z)=z+c, c$ constant.

Evidently $E$ cannot be a proper subset of any continuum of diameter 2 ; thus $E$ must be convex and, in fact, a complete set of diameter 2 [2, p. 122]. It is thus a set of constant width 2 [2, Theorem 52]. The boundary of a bounded closed convex plane set is rectifiable $[2$, p. 88] and for a set of constant width 2 the length is $2 \pi[2$, p. 127].

If the appropriate branch of $\left(f^{\prime}(z)\right)^{1 / 2}$ has about the point at infinity the development

$$
\left(f^{\prime}(z)\right)^{1 / 2}=1+\sum_{j=2}^{\infty} \beta_{j} z^{-j}
$$

the above length is given by the formula $2 \pi+2 \pi \sum_{j=2}^{\infty}\left|\beta_{j}\right|^{2}$. The corresponding formula for functions in $S$ [3] was given by Bieberbach [1]. The present formula can be found in [4]. (The latter paper may be somewhat difficult of access but in the present instance the formula is readily proved.) Thus we must have $\beta_{j}=0, j=2, \cdots$, and $f(z)$ $=z+c, c$ constant.

Received by the editors November 11, 1968. 


\section{BibliograPHy}

1. L. Bieberbach, Über die konforme Abbildung nahezu kreisförmiger Bereiche, S.-B. Preuss. Akad. Wiss. Berlin, Phys.-Math. Kl. (1924), 181-188.

2. H. G. Eggleston, Convexity, Cambridge Univ. Press, Cambridge, 1958.

3. James A. Jenkins, Univalent functions and conformal mapping, Springer-Verlag, Berlin, 1958.

4. Y. Komatu, $A$ variational problem in conformal mappings of multiply-connected regions, Rigaku 3 (1948), 3-6. (Japanese)

5. G. Pólya and G. Szegö, Aufgaben und Lehrsätze aus der Analysis. Vol. II, Springer, Berlin, 1925.

WASHINGTON UNIVERSITY 